\section{Commentary: Total aortic arch replacement and the frozen elephant trunk: Out with the old, in with the new?}

Erik Beckmann, MD, and Axel Haverich, MD

In this issue of the Journal, Ogino and colleagues ${ }^{1}$ present the early results of the Japanese multicenter study comparing conventional total aortic arch replacement with the frozen elephant trunk procedure using the Frozenix J-graft. Over a 3-year period, 684 patients underwent surgery with 1 of these operations. The early mortality was impressively low, as were the rates for most perioperative complications. Thus, the authors conclude that the frozen elephant trunk provides acceptable outcomes.

This study aimed to compare conventional arch replacement and frozen elephant trunk, but how successful can such a comparison be? Is it possible to deduce a "simply better" procedure? Obviously, the authors cannot answer simplistic questions like this, even though it would make life easier. Rather, they highlight advantages of the frozen elephant trunk and its drawbacks. The nuanced take: Wide application of the frozen elephant trunk is certainly justified by its low perioperative risks, but the traditional procedure remains relevant in modern practice for selected patients.

The frozen elephant trunk appears to benefit patients with acute aortic dissection by reexpansion of the true lumen immediately and stabilization of the distal aorta in the long term. ${ }^{2,3}$ In this study, the frozen elephant trunk was more commonly applied in patients with acute aortic dissection, supporting this idea. The authors focus exclusively on

\footnotetext{
From the Department of Cardiothoracic, Transplantation and Vascular Surgery, Hannover Medical School, Hannover, Germany.

Disclosures: The authors reported no conflicts of interest.

The Journal policy requires editors and reviewers to disclose conflicts of interest and to decline handling or reviewing manuscripts for which they may have a conflict of interest. The editors and reviewers of this article have no conflicts of interest.

Received for publication April 9, 2021; revisions received April 9, 2021; accepted for publication April 12, 2021; available ahead of print April 1, 2021.

Address for reprints: Axel Haverich, MD, Department of Cardiothoracic, Transplantation and Vascular Surgery, Hannover Medical School, Carl-Neuberg Str 1, 30625 Hannover, Germany (E-mail: haverich.axel@mh-hannover.de).

J Thorac Cardiovasc Surg 2022;164:1696-7 $0022-5223 / \$ 36.00$

Copyright $₫ 2021$ Published by Elsevier Inc. on behalf of The American Association for Thoracic Surgery

https://doi.org/10.1016/j.jtcvs.2021.04.028
}

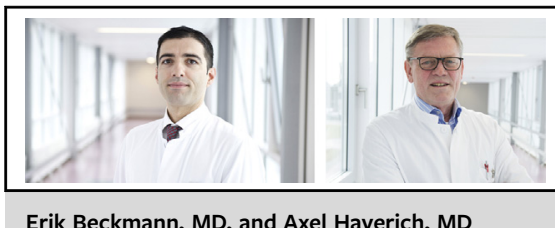

Erik Beckmann, MD, and Axel Haverich, MD

CENTRAL MESSAGE

The frozen elephant trunk procedure can be widely performed with extremely low perioperative risk.

short-term outcomes. Especially in the context of aortic dissection, the long-term outcomes of the frozen elephant trunk procedure are of great interest.

The relatively low but still-heightened risk of spinal cord ischemia is a potential disadvantage of the frozen elephant trunk compared with conventional arch replacement. ${ }^{4}$ In this study, the rate of paraplegia was $1.6 \%$ in the frozen elephant trunk group, whereas it was $0 \%$ in the conventional group. Such measures as cerebrospinal fluid drainage, proximalization of the distal anastomosis, and use of a short stent graft should be taken to reduce this risk. ${ }^{4}$ Furthermore, some patients' pathologies may not require frozen elephant trunk. Using conventional arch replacement instead avoids the risk of spinal cord injury completely. Thus, careful patient selection is important.

The study by Ogino and colleagues demonstrates today's widespread and extremely safe use of the frozen elephant trunk procedure in Japan. Even though the Frozenix J graft is not available in Europe and the United States, the results are of relevance, especially in the context of the upcoming introduction of the Thoraflex hybrid graft as the first commercially available hybrid graft in the United States. ${ }^{5}$ As the present study shows, the availability of off-theshelf solutions facilitates the spread of hybrid grafts, which in turn improves the safety of the operation. Practice makes perfect.

\section{References}

1. Ogino H, Okita Y, Uchida N, Kato M, Miyamoto S, Matsuda H, et al. Comparative study of Japanese frozen elephant trunk device for open aortic arch repairs. $J$ Thorac Cardiovasc Surg. 2022;164:1681-92.e2.

2. Shrestha M, Bachet J, Bavaria J, Carrel TP, De Paulis R, Di Bartolomeo R, et al. Current status and recommendations for use of the frozen elephant trunk technique: a position paper by the vascular domain of EACTS. Eur J Cardio-Thorac Surg. 2015;47:759-69. 
3. Chen Y, Ma W-G, Zhi A-H, Lu L, Zheng J, Zhang W, et al. Fate of distal aorta after frozen elephant trunk and total arch replacement for type A aortic dissection in Marfan syndrome. J Thorac Cardiovasc Surg. 2019;157:835-49.

4. Hagl C, Pichlmaier M, Khaladj N. Elephant trunks in aortic surgery: fresh and frozen. J Thorac Cardiovasc Surg. 2013;145:S98-102.
5. Shrestha M, Kaufeld T, Beckmann E, Fleissner F, Umminger J, Abd Alhadi F, et al. Total aortic arch replacement with a novel 4-branched frozen elephant trunk prosthesis: single-center results of the first 100 patients. J Thorac Cardiovasc Surg. 2016;152:148-59.e1. 